\title{
Construção de ambiente para desenvolvimento de jogos educacionais baseados em interface de gestos
}

\author{
João Roberto de T. Quadros ${ }^{1}$ \\ Rafael Castaneda ${ }^{1}$ \\ Myrna Amorim ${ }^{1}$ \\ Guilherme Herzog ${ }^{1}$ \\ Lucas Carneiro $^{1}$ \\ Kaique Menezes ${ }^{1}$ \\ Matheus Pinheiro ${ }^{1}$ \\ Daniel de Oliveira ${ }^{2}$ \\ Eduardo Ogasawara
}

\begin{abstract}
Resumo: Este artigo apresenta um projeto para a construção de um ambiente de programação de jogos educacionais baseado em interface de gestos, realizado dentro de uma escola federal de informática. $\mathrm{O}$ texto enfatiza a parte do projeto voltada para a montagem do ambiente por meio de equipamentos para a captura de movimentos e de uma plataforma de programação didática para construção de aplicativos. $\mathrm{O}$ artigo, também, demonstra o desenvolvimento de protótipos visando ao teste desse ambiente, de modo a prepará-lo para que sejam desenvolvidos aplicativos mais complexos voltados para a área educacional.
\end{abstract}

Palavras-chave: Informática na educação, jogos computacionais, Interface homem-máquina.

\begin{abstract}
This paper presents a project to build a programming environment for educational games based on motion interface that was developed within a federal school of computer science. This paper emphasizes the development of the environment through motion capture equipment and programming platform for development of applications based on didactics principals. It also presents the development of prototypes to test this environment and prepare it for the development of more complex applications targeting the educational field.
\end{abstract}

Keywords: Educational computing.

\section{Instruções gerais}

Dominar e trabalhar com os fundamentos da programação de computadores é uma das etapas mais difíceis para estudantes de cursos de computação. Essa limitação tem motivado os professores a criarem e usarem diversas ferramentas que tornem o ensino de programação mais intuitivo e que produzam aplicativos úteis para a sociedade.

Uma ideia surgida nos cursos da Escola de Informática do Centro Federal de Educação Tecnológica Celso Suckow da Fonseca (CEFET/RJ) foi a de se introduzir um ambiente especializado para a criação de jogos de computador que contenham características motivacionais associadas ao processo de ensino-aprendizagem que vão além dos métodos tradicionais de ensino. Nela, os alunos vão poder criar jogos e programas por meio de uma plataforma de desenvolvimento que combine as tecnologias de realidade virtual, tais como as interfaces de gestos. Essa abordagem é direcionada para duas vertentes: tornar o ato de programar mais atraente, diminuindo a resistência em seu aprendizado, e auxiliar na produção de aplicativos

\footnotetext{
${ }^{1}$ Escola de Informática, CEFET/RJ, Av. Maracanã, 229, pavilhão \#1 - Maracanã - Rio de Janeiro (RJ) - Brasil

http://dx.doi.org/10.5335/rbca.2013.3270 
úteis no campo do ensino, tais como jogos interativos que trabalhem com a percepção cognitiva associada a movimento e ações.

Os sistemas construídos com capacidade de interação homem-máquina (IHM) mais cognitiva implementam funcionalidades que servem para criar situações e ambientes que facilitam tarefas de aprendizagem. Podem atuar a partir da manipulação de tecnologias de realidade virtual em tarefas de ensino, tais como compreensão da mecânica de movimentos em geral, aperfeiçoamento das características sobre como trabalhar o corpo, interação completa e em tempo real, como as de mundos de regras específicas e diferentes, nas quais se possa, dentre outras atividades, captar e perceber novas sensações e prover ambientações dirigidas às deficiências específicas associadas às dificuldades de movimentos.

Neste trabalho, o objetivo é apresentar uma parte do projeto, ainda em andamento, que envolve a construção e o uso de um ambiente de desenvolvimento de aplicativos baseados na interface de gestos Kinect, com auxílio da plataforma de desenvolvimento Greenfoot. Esse ambiente está sendo consolidado para que a tarefa de programar se torne mais fácil e também para que os alunos da Escola de Informática possam construir sistemas com um grau de interatividade complexo, nos quais, na evolução de suas funcionalidades, passe a ser possível a produção de sistemas de apoio à educação e ao ensino.

São verificados alguns resultados iniciais, na forma de protótipos simples feitos nesse ambiente, que utilizam Applications Programming Interfaces (APIs), com código aberto e reutilizável, já existentes e oferecidas pelo fornecedor do Kinect e que foram adaptadas no ambiente de programação Greenfoot para tratar da parte de interface de gestos. Esses protótipos têm servido de base no aprendizado desse ambiente, facilitando a compreensão desse tipo de sistema, de modo que a próxima fase do projeto, que envolve o desenvolvimento de jogos educacionais, aplicações na área de inteligência artificial e simuladores, seja também iniciada.

Além desta introdução, o artigo é dividido em mais cinco seções. Na seção 2, é apresentada a Escola de Informática do CEFET/RJ. Na seção 3, expõe-se a proposta do uso de jogos como ferramenta pedagógica. A seção 4 aborda alguns aspectos da IHM. A seção 5, por sua vez, tem como objeto o ambiente de jogos e seu uso associado à interface de gestos na Escola de Informática. Concluindo o artigo, a seção 6 elenca os resultados alcançados e pontos a serem explorados.

\section{A Escola de Informática do CEFET/RJ}

A Escola de Informática do CEFET/RJ constitui-se pelos cursos de Técnico de Informática, curso Superior de Tecnologia em Sistemas para Internet (CST-SI) e Bacharelado em Ciência da Computação (BCC). O curso Técnico de Informática foi criado em 2000; o CST-SI, em 2002; e o BCC iniciou sua primeira turma no segundo semestre de 2012. Todos os cursos abordados neste artigo são oferecidos na unidade Maracanã do CEFET/RJ.

O curso Técnico de Informática é um curso de três anos, semestral, com 1.260 horas-aula e 400 horas de estágio. É um curso concomitante ao ensino médio e frequentado por alunos que têm entre 13 e 18 anos de idade. Possui 20 disciplinas, dentre as quais, cerca de 85\% trabalham com aulas prática, e destas, ainda, 70\% são associadas à programação, análise e desenvolvimento de sistemas. $\mathrm{O}$ curso também aborda outros fundamentos da área de informática, tais como hardware e arquitetura de computadores, sistemas operacionais, redes de computadores, engenharia de software e banco de dados. O CST-SI foi criado como um curso com duração mínima de 6 e máxima de 9 semestres. É um curso de graduação tecnológica com ênfase em desenvolvimento de sistemas de software para o ambiente da Internet, com oferecimento de disciplinas específicas da área, como tecnologias Web, linguagens de programação, engenharia de software, redes de computadores, bancos de dados, arquitetura de computadores e sistemas operacionais, e outras de cunho mais geral, tais como informática na sociedade e empreendedorismo. O BCC foi criado em 2012, sendo um curso de graduação plena com ênfase na formação de cientistas da área de computação. Está organizado em 8 semestres, com duração máxima de 12 semestres. Possui muitas disciplinas em comum com o curso CST-SI, mas também com disciplinas diferenciadas, voltadas para o aprimoramento científico, tais como: inteligência computacional, jogos computacionais, prática de pesquisa aplicada, grafos, matemática básica e avançada, entre outras. Seu foco é atuar diretamente nas linhas de pesquisa do Grupo de Pesquisa em Computação Aplicada (GPCA) da Escola de Informática visando, mais especificamente, às áreas de jogos educacionais e inteligência computacional.

As noções básicas de programação e estrutura de dados são ministradas nos três primeiros períodos de cada curso, de forma a alcançar a solidificação dos conceitos de confecção de sistemas. Nos períodos 
subsequentes, avança-se mais em desenvolvimento de sistemas, uma vez que os alunos adquirem conhecimento em linguagens de programação mais sofisticadas e, utilizando os conceitos obtidos, começam a tratar das características práticas associadas ao projeto de sistemas computacionais e aplicativos.

Apesar de existir um curso diretamente voltado para a pesquisa científica (o BCC), todos os demais contribuem no desenvolvimento das pesquisas do grupo de pesquisa, existindo, assim, a participação, também, de alunos do Técnico de Informática e do CST-SI. Além disso, há o incentivo para participações de diversos eventos internos e externos, entre os quais, a Olimpíada Brasileira de Informática [16], Maratona de Programação da Universidade Estadual do Rio de Janeiro, tendo os alunos dos cursos obtido bons resultados.

A entrada em todos os cursos é via concurso público. No caso do Técnico de Informática, o ingresso se dá por meio de concurso público comum, realizado em data e local determinados por edital, e no caso dos cursos superiores (CST-SI e BCC), pelo Enem e matrícula no Sisu. Todos os ingressos nos cursos estão de acordo com a Lei 12.711/2012, que estabelece regras de ação positiva e entrada por cotas, para cumprir seu papel social conforme as diretrizes do governo federal.

\section{Uso da tecnologia de jogos eletrônicos como ferramenta pedagógica}

Áreas como Gestão Empresarial $[6,8,11]$ e Educação $[4,12,17]$ vêm fazendo o uso de ferramentas baseadas em jogos eletrônicos para o auxílio de suas metodologias pedagógicas. Na gestão empresarial, os jogos são usados para a compreensão de aspectos de negociação e administração de empresas, por meio de simulações de situações que contenham aspectos decisórios e competitivos $[6,11]$. Na educação, os jogos servem para auxiliar a compreensão de aspectos práticos de diversas disciplinas, tais como matemática, biologia, química e física $[4,12,17]$. Jogos também têm sido desenvolvidos para auxiliar no reforço de aprendizagem de pacientes portadores de deficiências cognitivas [1,13].

Segundo Tarouco et al. [20], os jogos tornam-se ferramentas eficientes para esse fim, pois contêm elementos de diversão, motivação, além de facilitarem o aprendizado e aumentarem a capacidade de retenção do conteúdo ensinado. Jogos eletrônicos transformam-se, assim, em uma excelente ferramenta de ensino, na medida em que atuam sobre as funções mentais e intelectuais do jogador e contribuem nos elementos de didática de ensino. Tarouco [21] atribui a atração sobre esse tipo de ferramenta às características dessas modalidades de jogos, tais como uso de regras e estruturas bem elaboradas, metas e objetivos motivacionais, interação, que permitem ação e reação dos usuários, trabalho com resultados e realimentação de conteúdo, facilitando a aprendizagem de conceitos, e, acima de tudo, ao aspecto da diversão, tornando o processo de aprendizado mais agradável.

Os jogos não são vistos por quem os desenvolve apenas como um modo de entretenimento. Em trabalhos como o de Johnson [7], os jogos eletrônicos são apresentados como ferramentas que auxiliam o aluno a decidir, a escolher, a priorizar, de acordo com regras e limites existentes, trazendo benefícios intelectuais. Conforme o autor [7], tais recursos permitem a construção de métodos para aprender a pensar e a tomar a decisão certa sobre o peso de evidências e da análise de situações, podendo auxiliar na definição de metas de longo prazo.

Se, por um lado, jogar facilita o aprendizado, por outro, no contexto de computação, desenvolver um jogo é um elemento motivacional de aprendizado para quem o programa. Um dos desafios em questão é, ao mesmo tempo, aprender os conceitos de programação e produzir jogos interessantes em um ambiente que não seja complexo demais para não desestimular o aprendizado. Nesse contexto de facilitar o aprendizado de programação, existem algumas iniciativas, dentre as quais se pode destacar o Greenfoot, que é uma ferramenta de software concebida para permitir que iniciantes adquiram experiência com programação orientada ao objeto. Ele suporta o desenvolvimento de aplicações gráficas na linguagem de programação Java. O Greenfoot foi concebido e implementado na Universidade de Kent, Inglaterra e na Universidade Deakin, Melbourne, Austrália, em 2006, sob a supervisão de Michael Kölling [9].

O ambiente de programação do Greenfoot é integrado e inclui capacidade de gerir vários projetos ao mesmo tempo, editar, depurar a sintaxe e executar aplicativos. Essa interface foi construída para ser de uso simples e, ao mesmo tempo, permitir a transição para outros ambientes de programação. $\mathrm{O}$ modelo de programação Greenfoot consiste no uso de uma classe World (representado por uma área de tela retangular) e um conjunto de objetos da classe Actor que estão presentes no World e podem ser programados para atuar de forma independente. Tanto World quanto Actor representam classes e objetos específicos de Java. O Greenfoot oferece vários métodos padrões de programação, tais como tratamento de rotação, movimento, mudanças de aparência e 
detecção de colisão. A Figura 1 apresenta um exemplo do desenvolvimento de um jogo baseado no modelo de Lotka-Volterra $[2,5,19]$. Note a especialização Worldfc da classe World para representar o jogo propriamente dito e as especializações Chicken e Fox referentes à classe Actor.

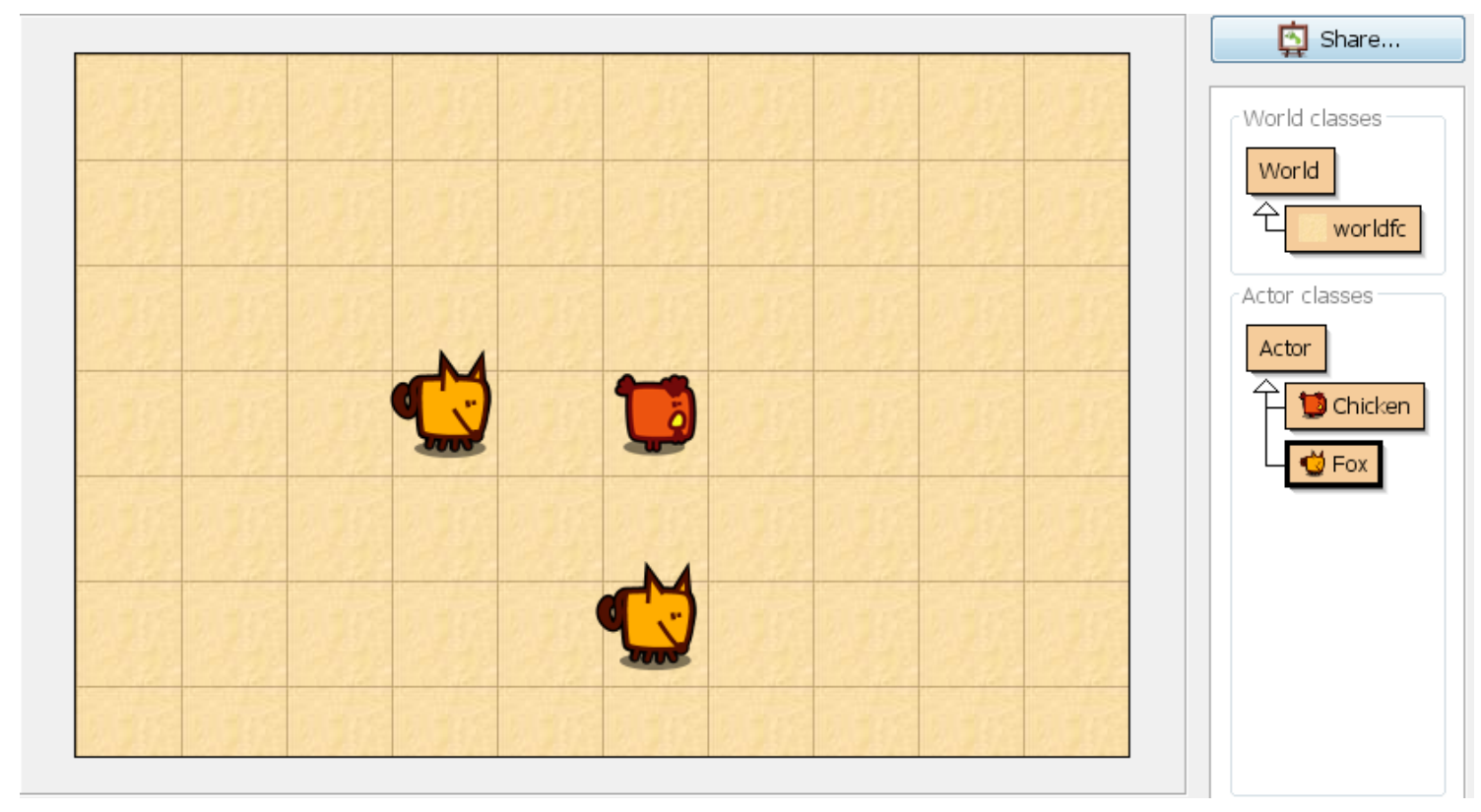

Figura 1 - Exemplo da elaboração de um jogo baseado em Lotka Volterra [19] usando Greenfoot

\section{Tecnologia de interface homem-máquina e uso de interface de gestos}

IHM é uma área multidisciplinar que envolve as áreas de ciência da computação, psicologia, linguística, artes, dentre outras [15, 24]. O conhecimento sobre as limitações da capacidade humana, restrições e evoluções (em termos de dispositivos, interfaces e poder de processamento) das tecnologias existentes devem ser levados em conta para oferecer aos usuários uma forma adequada para interagir com plataformas computacionais.

Uma das abordagens tecnológicas que envolvem os conceitos dessa área é a utilização de ambientes de realidade virtual que ajudem a simular situações e os aspectos cognitivos da IHM [18]. A montagem de um ambiente que trate de realidade virtual vai exigir equipamentos e software que atuem com os objetivos dessa tecnologia. Entre os mecanismos e tecnologias que focam em realidade virtual existem as interfaces de gestos.

A interface de gestos é uma tecnologia IHM que visa a apresentar e recriar ao máximo a sensação de realidade para um indivíduo. Faz-se uso de sensores e software para montar um mundo virtual onde se possa interpretar movimentos e sensações. Esse tipo de interface é vista como um capturador da realidade por meio de recursos computacionais, direcionando atuações sobre características temporais [10]. Desse modo, as interações devem ocorrer com os equipamentos computacionais em tempo real, assim como as respostas das operações executadas pelo usuário. Entre as ações que devem ser capturadas e tratadas em tempo real estão: movimentos corporais, captação da voz e do campo visual do operador [10]. Essas interfaces também podem traduzir os eventos captados no mundo real e adaptá-los a universos não propriamente reais, seja por ícones, símbolos ou regras diferentes das daquele contexto, criando um mundo ficcional, onde se podem experimentar sensações não percebidas no mundo real, como, por exemplo, voar [10]. Essa abordagem foca o trabalho com reações, cognição e relações intelectuais voltadas para a compreensão e as interações com sensações.

A evolução recente das tecnologias para tratamento de interações dos usuários, muitas vezes de forma pervasiva, criou novos paradigmas para a concepção de interfaces gestuais para aplicações interativas, como o Kinect [22], que é um sensor ou capturador de movimento. Esse equipamento é um ícone comercial caracterizador dessa nova forma de comunicar-se com o usuário e que se presta a auxiliar na comunicação por meio de interações naturais. Os sensores de movimento são equipamentos modernos e servem para capturar os movimentos de usuários sem a necessidade de controles manuais, com a finalidade, por exemplo, de comandar 
as ações de um jogo ou aplicativo de software, tornando os jogos mais inovadores e atrativos, além de fornecer níveis mais apropriados de usabilidade e acessibilidade para usuários com limitações físicas.

Uma visualização do Kinect visto é apresentada na Figura 2. Por ser uma interface de gestos, baseia-se em sensor de movimentos, que trabalha em três dimensões e é capaz de rastrear diversos usuários, identificando individualmente gestos e posições. O sensor é composto de três partes para a captação dos movimentos e da voz do usuário:

- Câmera de vídeo VGA colorida - auxilia no reconhecimento facial e na detecção de outras características, ao evidenciar três cores componentes: vermelho, verde e azul.

- Projetor IR e câmera IR - o projetor infravermelho aliado à câmera infravermelha funciona de modo combinado como um sensor de profundidade. Eles trabalham juntos para "ver" a sala em 3D, possibilitando ao Kinect ter uma noção de profundidade sem levar em consideração as condições de iluminação.

- Microfone multimatriz - é um dos quatro microfones que podem isolar as vozes dos jogadores do barulho da sala. Possibilita ao jogador estar a poucos metros de distância do microfone e, ainda, usar controles por voz.

Além do uso com o console, o Kinect pode ser conectado diretamente a um computador, como mecanismo de interface humano-computador em jogos e aplicativos, acessível por linguagens de programação como Java e C++. Ademais, o Greenfoot também oferece classes específicas para programar o Kinect dentro do ambiente.

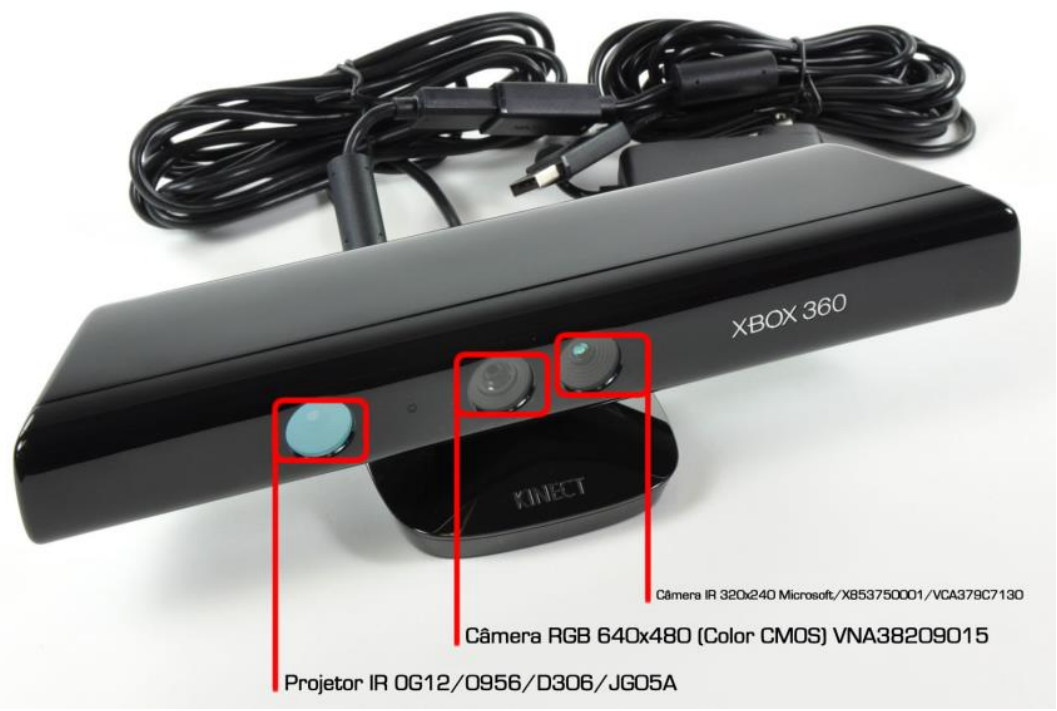

Figura 2 - Exemplo do equipamento Kinect [14]

\section{Ambiente de jogos com uso com interface de gestos no CEFET/RJ}

Em 2011, iniciou-se o projeto de construção do ambiente para desenvolvimento de jogos e aplicativos que fizessem uso de uma interface de gestos. Escolheu-se, para tanto, o Kinect, em razão do conhecimento prévio dos participantes do grupo de pesquisa acerca desse equipamento e pelo fato de este ter uma interface com a plataforma Greenfoot, já em uso nas disciplinas dos cursos da Escola de Informática. A partir desse momento, foram obtidos, por meio do apoio de órgãos de fomento, dentre os quais, a Coordenação de Aperfeiçoamento de Pessoal de Nível Superior (Capes) e a Fundação Carlos Chagas Filho de Amparo à Pesquisa do Estado do Rio de Janeiro (FAPERJ), dois equipamentos Kinect, um XBox360, o pacote de bibliotecas de desenvolvimento, que os acompanha, e computadores de apoio para montagem de um laboratório de pesquisa, além de bolsas Pibic e Pibic-EM oferecidas pelo CNPq e pelo CEFET/RJ. 
A elaboração dos primeiros protótipos, a partir de adaptações de APIs do Kinect presentes no Greenfoot, representou o marco inicial do projeto, que, nessa fase, envolveu um professor do grupo de pesquisa e um aluno do curso Técnico de Informática, servindo de base para que esses participantes pudessem aprimorar o desenvolvimento da ferramenta e estudar melhor suas características. As conclusões e os aprendizados obtidos foram repassados para os demais membros do grupo de pesquisa.

Como resultado imediato desse estudo, já em outubro de 2011, houve uma apresentação, na Feira de Tecnologia do CEFET/RJ (EXPOTEC), de dois jogos, que trabalharam com a captação de movimentos. O primeiro jogo foi o Pong, inspirado no jogo de Paredão do Telejogo II [23]. A diferença principal diz respeito ao fato de o controle dos jogadores ter sido feito a partir de seus movimentos, ao contrário das manetes do Telejogo. A Figura 3 apresenta o jogo e dois alunos controlando os jogadores do Pong. Ao fazer uso do Greenfoot com a API do Kinect, foi possível observar que a implementação do controle do jogo não foi complexa, conforme pode ser visto na Figura 4.

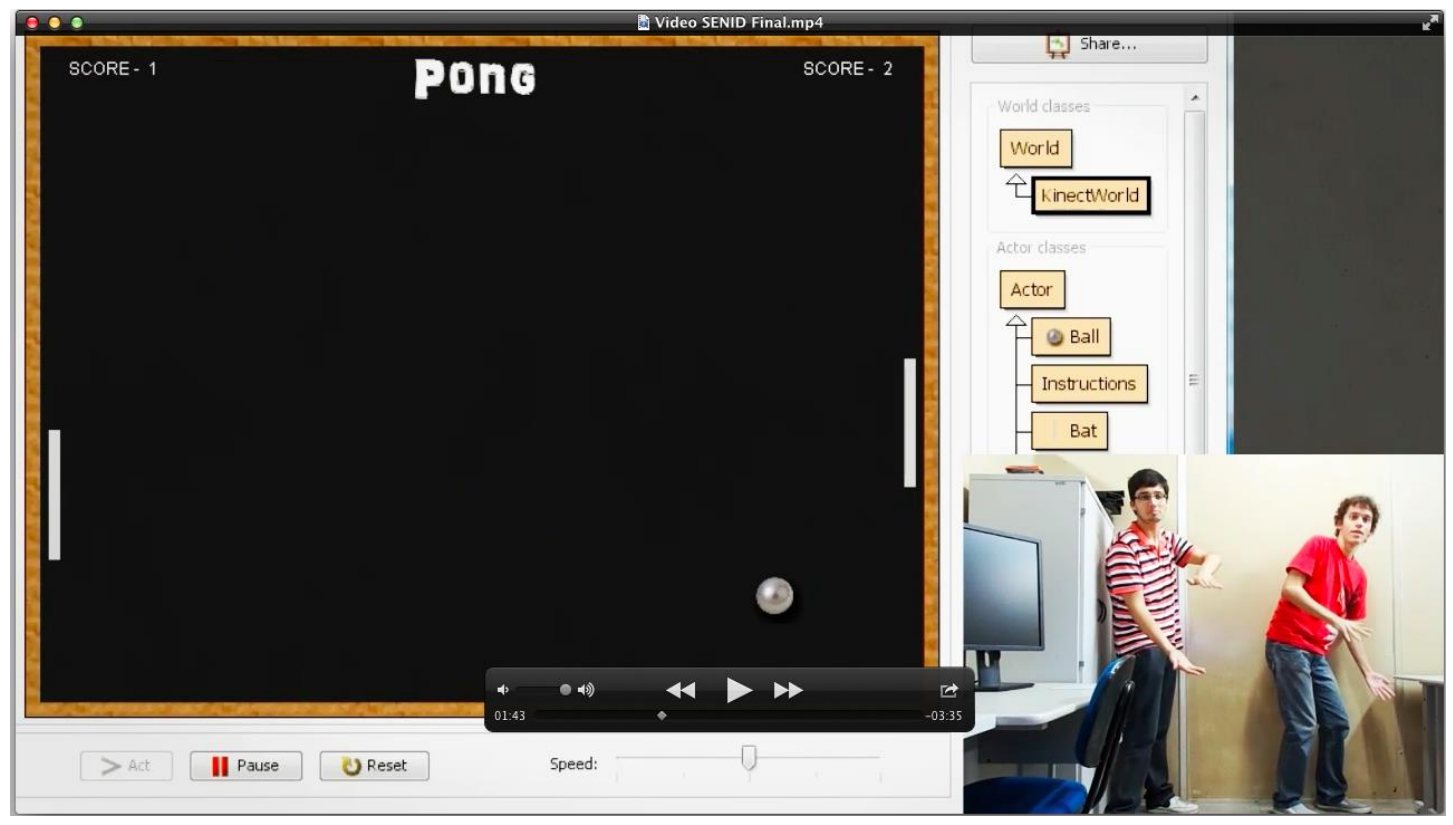

Figura 3 - Alunos fazendo uso do protótipo do Pong

Com os resultados iniciais do Pong, também foi possível desenvolver uma versão inicial de um segundo jogo mais sofisticado. O jogo funcionava de modo a permitir que o usuário, usando os braços e pés, capturasse bolas que caíssem da tela. Os movimentos desses membros eram traduzidos via Kinect para uma interface visual (uma tela própria) na qual as bolas que caíssem e cruzassem as linhas na interface que identificassem as mãos e os pés do usuário seriam aparadas na tela, como se o usuário as estivesse segurando no mundo real. Um exemplo do uso desse protótipo pode ser visto na Figura 5.

Esses dois modelos geraram um significativo entusiasmo entre os alunos dos cursos da Escola de Informática. Foram promovidos pequenos workshops para os interessados em participar dos projetos envolvendo essa ferramenta, e, por meio do incentivo da Bolsa de Pesquisa CEFET/RJ, outros projetos com o uso dessa ferramenta foram criados. Já no início de 2012, houve o envolvimento de mais dois professores do grupo de pesquisa e de três alunos bolsistas da Escola de Informática (dois do Técnico de Informática e um do CST-SI), além de outros alunos que trabalhavam como voluntários na construção de outros sistemas baseados em interface de gestos. 


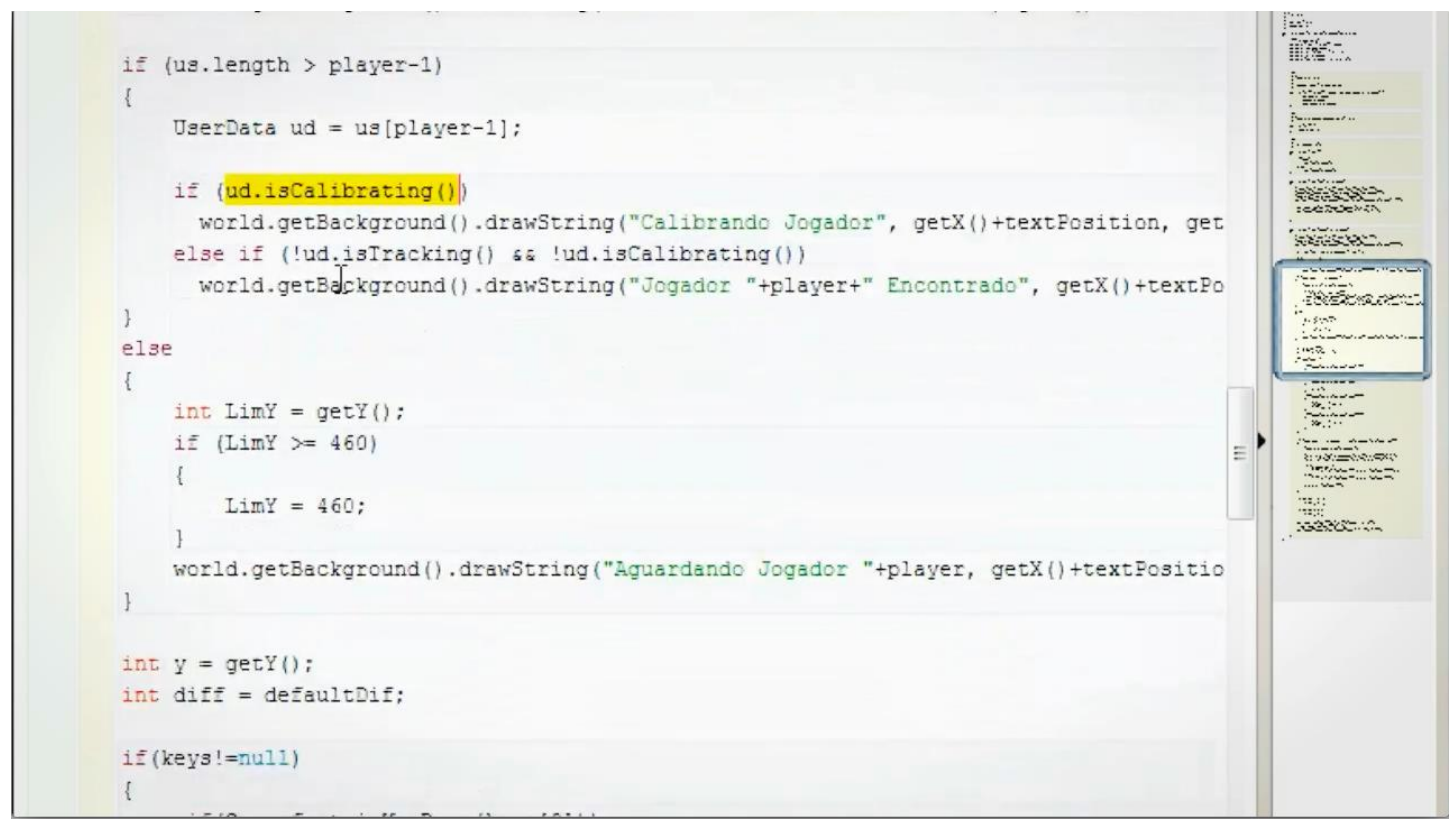

Figura 4 - Código do protótipo do Pong

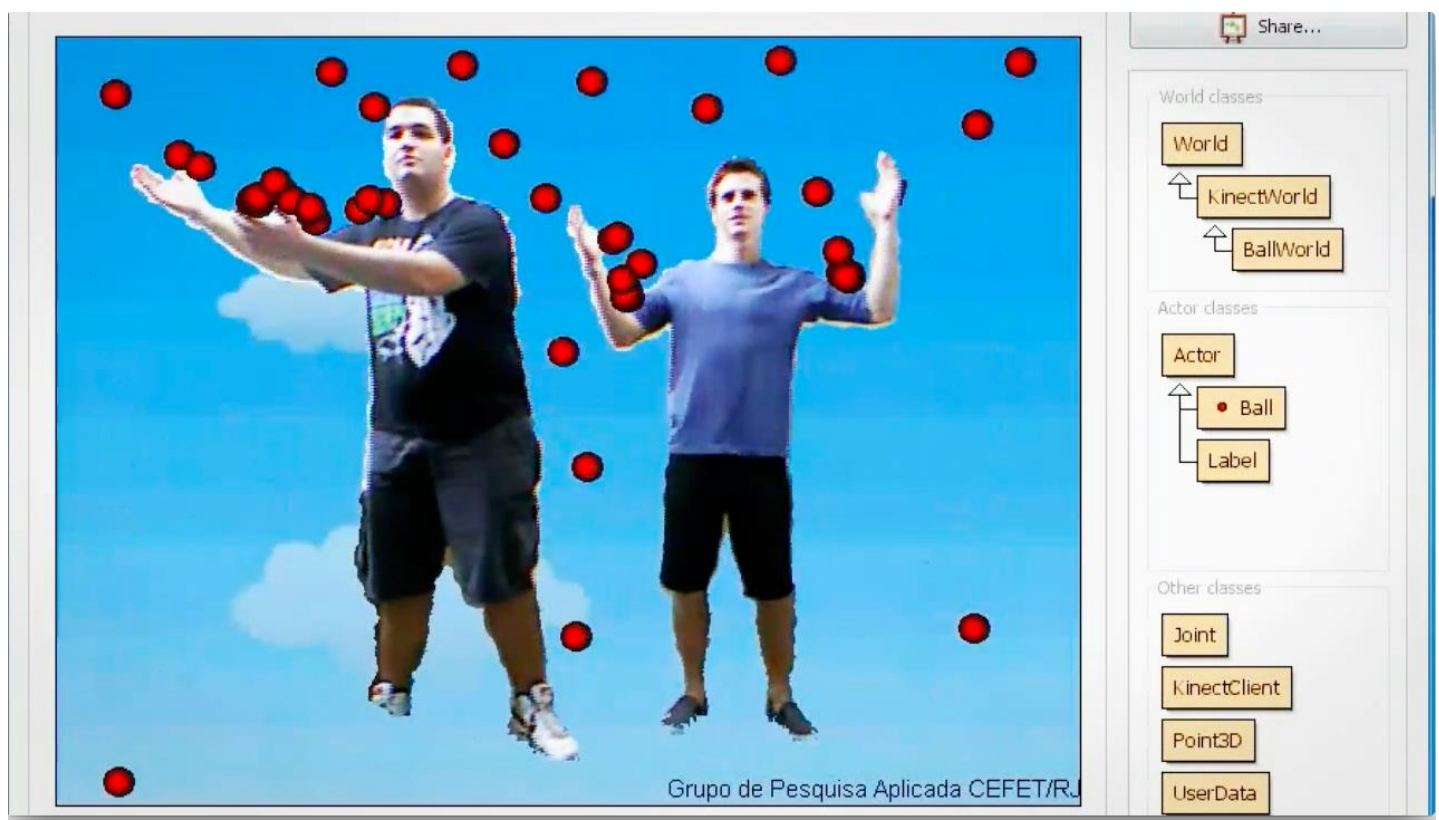

Figura 5 - Alunos experimentando o protótipo de captura de bolas

Em 2012, foram dados mais alguns passos no desenvolvimento de aplicativos com interface de gestos. Deu-se ênfase a projetos voltados para desenvolver aplicativos que pudessem contribuir com as áreas de educação e ensino. Como resultado dessa abordagem foram aproveitadas mais APIs existentes na plataforma para se desenvolver um aplicativo de captura dos gestos - no caso, de gestos da mão de um usuário -, de modo que esse usuário pudesse simular estar escrevendo em uma tela imaginária, uma espécie de quadro virtual. Nesse ambiente, a proposta era que um aluno, ao escrever, pudesse ter seus textos verificados e corrigidos por um professor. Foi identificado, também, que esse protótipo poderia ser uma base para um aplicativo de ajuda a pessoas com deficiências motoras que desejassem comunicar-se com gestos. Um exemplo desse aplicativo em uso pode ser visto na Figura 6 (calibração do usuário para utilizar o quadro virtual) e na Figura 7 (utilização do quadro virtual propriamente dito). 


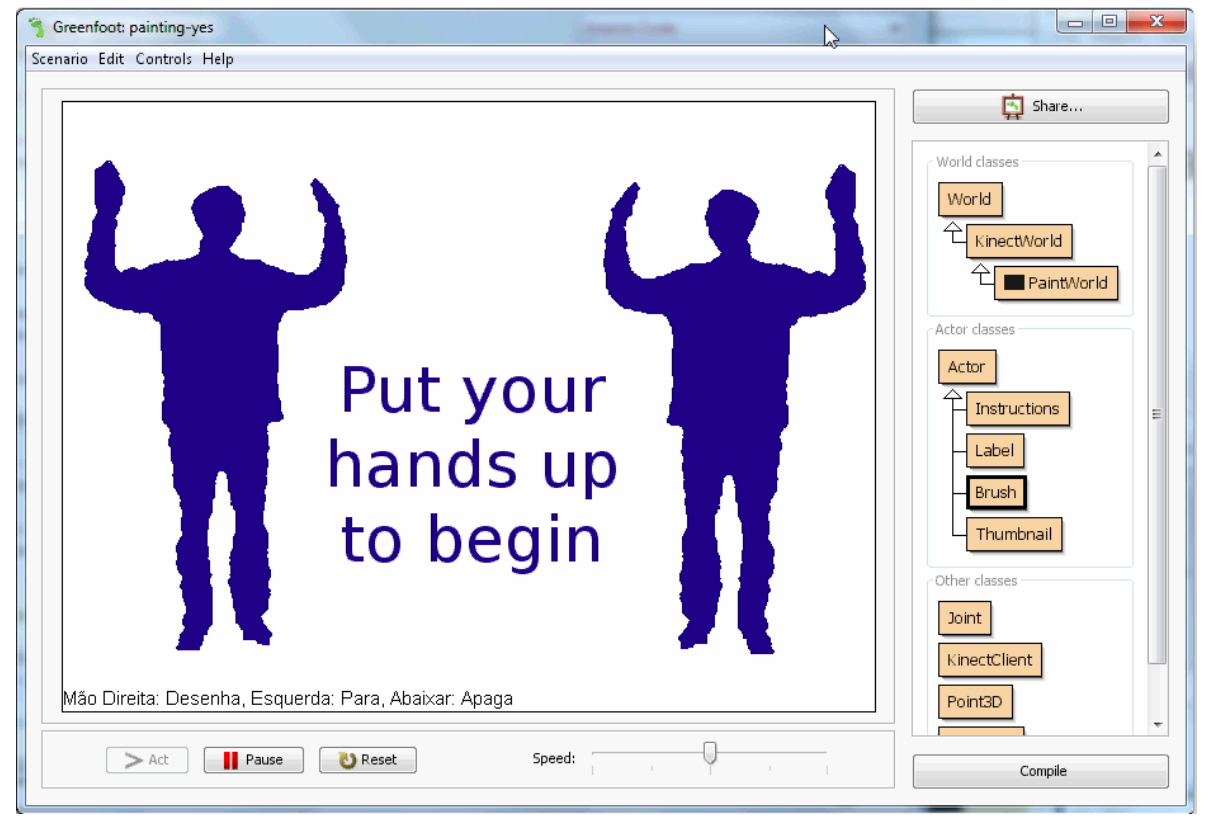

Figura 6 - Protótipo de quadro virtual

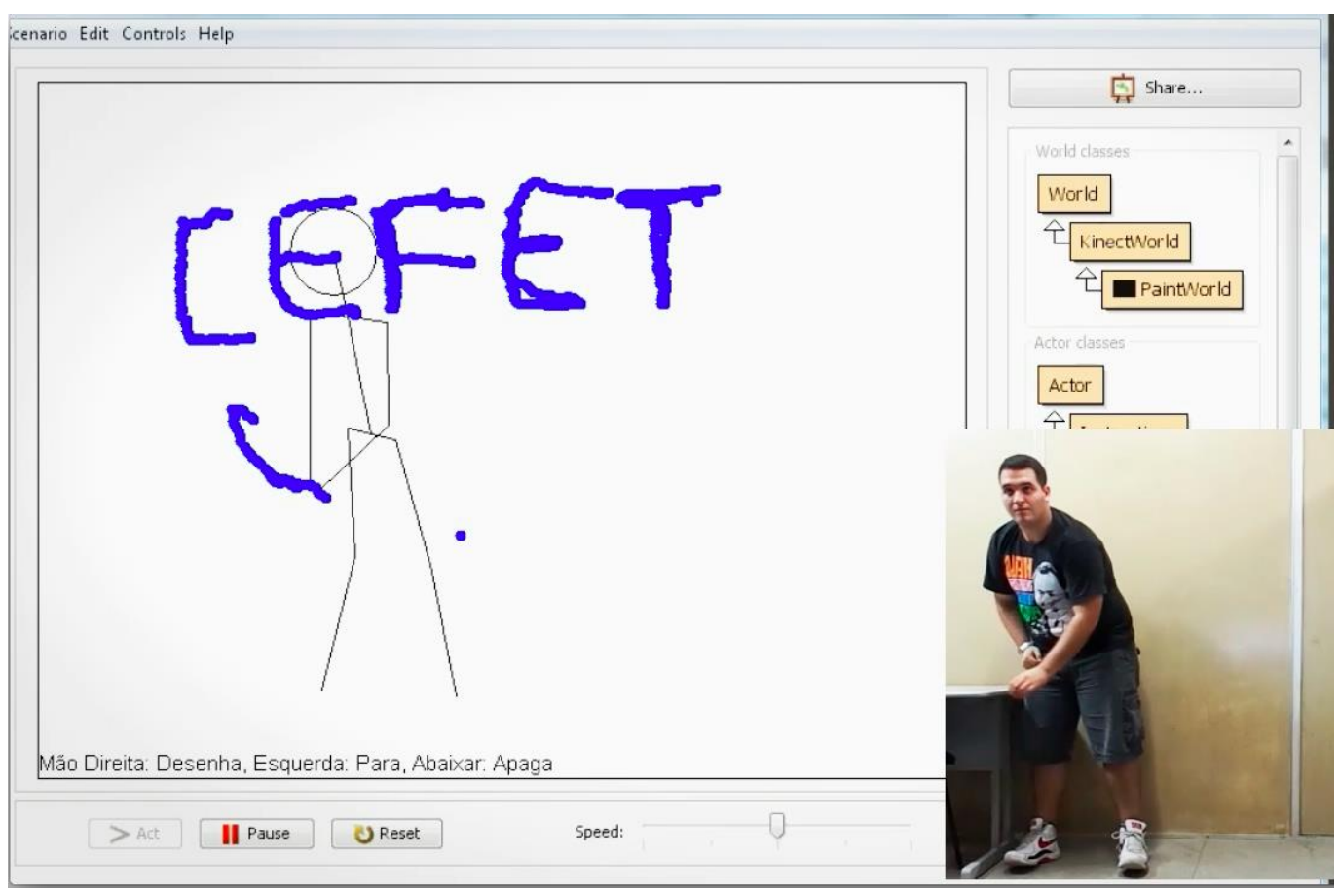

Figura 7 - Aluno usando o aplicativo do quadro virtual

Outro protótipo desenvolvido foi um sistema para captar e gravar movimentos de dança, de modo que pudessem ser reexibidos para uma futura análise. A ideia básica do aplicativo desenvolvido foi tornar possível a captura, gravação e análise de imagens representativas de movimentos de dança, usando uma das APIs que 
acompanham o Kinect denominada de BodyGuard. Esse aplicativo permitiria que um professor da disciplina de artes, por exemplo, analisasse os movimentos gravados, propondo acertos na coreografia feita pelo aluno.

Novamente, ambos os jogos, juntamente com as evoluções dos jogos de Pong e de captura de bolas, foram apresentados na EXPOTEC 2012. O resultado dessa apresentação pode ser conferido no vídeo utilizado durante a exposição do presente trabalho no SENID 2013 [3].

\section{Conclusão}

O projeto para criar um ambiente de desenvolvimento de jogos baseado em interface de gestos caminha para sua fase de maturação. Os pequenos aplicativos desenvolvidos serviram para que as equipes pudessem conhecer as ferramentas envolvidas nas produções dos aplicativos, melhorando o entendimento de suas características e peculiaridades. Além disso, esses protótipos fomentaram entre os alunos da Escola de Informática uma maior motivação no que tange às disciplinas de programação e, também, à procura de bolsas de incentivo à pesquisa oferecidas pelo próprio CEFET/RJ. Isso está possibilitando passar para a próxima fase do projeto, que é a construção de produtos mais complexos voltados para o apoio à educação.

Há vários alvos a serem focados, tais como: desenvolvimento de ferramentas de apoio a disciplinas do ensino médio-técnico, apoio ao ensino de pessoas portadoras de pequenas deficiências motoras e o desenvolvimento de jogos educacionais para o público infantil. Espera-se que, a partir da montagem dessa estrutura de pesquisa e dos estudos iniciados sob sua base, isso se torne possível.

\section{Agradecimentos}

Os autores, bem como o GPCA da Escola de Informática do CEFET/RJ agradecem aos órgãos de fomento FAPERJ, Capes e CNPq por sua contribuição nessa pesquisa. Ao mesmo tempo, o grupo agradece ao próprio CEFET/RJ pelo incentivo constante.

\section{Referências}

[1] FARIAS, A.A.R.; BARROS, M.L.N.L. O jogo como recurso psicopedagógico no atendimento do aluno com deficiência intelectual. Disponível em: $<$ http://www.psicopedagogiabrasil.com.br/artigos_alcina_ojogo.htm>. Acesso em: 02 mai. 2013. 2009.

[2] FLAKE, G.W. The Computational Beauty of Nature: Computer Explorations of Fractals, Chaos, Complex Systems, and Adaptation. Cambridge, MA, USA. The MIT Press. 2000.

[3] GPCA 2013. Video sobre Construção de Ambiente para Desenvolvimento de Jogos Educacionais Baseados em Interface de Gestos. Disponível em: <http://coinfo.cefet-rj.br/wp-content/uploads/2013/04/VideoSENID-Final.mp4>. Acesso em: 02 mai. 2013.

[4] GRANDO, R.C.; LORENZATO, S.A. O jogo [e] suas possibilidades metodologicas no processo ensinoaprendizagem da matematica. Unicamp - Faculdade de Educação - FE. 1995

[5] HOPPENSTEADT, F. Predator-prey model. Scholarpedia. v. 1, n.10, p.1563, 2006.

[6] IBM. INNOV8 $2.0 \quad-\quad A \quad B P M \quad$ Simulator. Disponível em: $\quad<$ http://www01.ibm.com/software/solutions/soa/innov8/index.html>. Acesso em: 02 mai. 2013.

[7] JOHNSON, S. Surpreendente!: a televisão e o videogame nos tornam mais inteligentes. Campus - BB. 2005.

[8] KALLAS, D. Balanced scorecard: aplicação e impactos: um estudo com jogos de empresas. USP Faculdade de Economia, Administração e Contabilidade. 2013. 
[9] KÖLLING, M. Introduction to programming with Greenfoot: object-oriented programming in Java with games and simulations. Prentice Hall. 2010.

[10] KORTUM, P. HCI beyond the GUI design for haptic, speech, olfactory and other nontraditional interfaces. Elsevier/Morgan Kaufmann. 2008.

[11] LEPSCH, S.L.; HEMZO, M.A. Jogos de Empresas com Foco em Marketing Estratégico: uma Análise Fatorial da Percepção dos Participantes. Revista Brasileira de Gestão de Negócios. v. 8, n.20, p. 23-33, 2006.

[12] LUCENA, M. O uso das tecnologias da informática para o desenvolvimento da educação. Publicação Técnicas. Rio de Janeiro: COPPE Sistemas/UFRJ, jul 1994. (ES-301/94). 1994.

[13] MAFRA, S.R.C. O Lúdico e o Desenvolvimento da Criança Deficiente Intelectual. Disponível em: $<$ http://www.diaadiaeducacao.pr.gov.br/portals/pde/arquivos/2444-6.pdf>. Acesso em: 02 mai. 2013. 2008.

[14] MICROSOFT. Kinect for Windows. Disponível em: <http://www.microsoft.com/en-us/kinectforwindows>. Acesso em: 02 mai. 2013.

[15] NETO, A.R. et al. Interfaces para aplicações de Interação Natural baseadas na API OpenNI e na Plataforma Kinect. Minicursos do WebMedia 2011. SBC. Florianópolis, SC, Brasil. 2011.

[16] OBI. Olimpiada Brasileira de Informática. Disponível em: <http://olimpiada.ic.unicamp.br>. Acesso em: 02 mai. 2013.

[17] OVERMARS, M.H. Game design in education. Institute of Information and Computing Sciences, Utrecht University. 2004

[18] PIMENTA, F.J.P. O Conceito de Virtualização de Pierre Levy e sua Aplicação em Hipermídia. LuminaFACOM//UPFJF. v. 4, n.1, p. 85-96, 2001.

[19] SCHOCAIR, C. et al. Estudo de Caso do Uso de Jogos Eletrônicos na Ministração de conceitos pedagógicos: simulação espacial do modelo Presa-Predador de Lotka-Volterra. Simpósio de Inovação Tecnológica na Educação (Campinas, São Paulo, Brazil, 2012), 1-14. 2012.

[20] TAROUCO, L.M.R. et al. Jogos Educacionais. Novas Tecnologias CINTED-UFRGS na Educação. v. 2, n.1, p. 1-7, 2004.

[21] TAROUCO, L.M.R. Jogos, Computador e Internet na Educação. Disponível em: $<$ http://penta3.ufrgs.br/animacoes/JogosEducacionais>. Acesso em: 02 mai. 2013.

[22] WIKIPEDIA. Kinect. Disponível em: <http://en.wikipedia.org/wiki/Kinect>. Acesso em: 02 mai. 2013.

[23] WIKIPEDIA. Telejogo II. Disponível em: <http://pt.wikipedia.org/wiki/Telejogo_II>. Acesso em: 02 mai. 2013.

[24] YONCK, R. The age of the interface: from processing codes punched out on cards to interpreting our brain waves, our computers are progressively learning how to ... better.(Essay): The Futurist. v. 44, n.3, 2010. 